\title{
Microfluidic time-division multiplexing accessing (TDMA) resistive pulse sensor for particle analysis
}

\author{
Gihoon Choi ${ }^{\mathrm{a} \dagger}$, Erica Murphy ${ }^{\mathrm{a} \dagger}$, and Weihua Guan ${ }^{\mathrm{a}, \mathrm{b}^{*}}$ \\ ${ }^{a}$ Department of Electrical Engineering, Pennsylvania State University, University Park 16802, USA \\ ${ }^{\mathrm{b}}$ Department of Biomedical Engineering, Pennsylvania State University, University Park 16802, USA \\ $\uparrow$ These authors contributed equally to this work. \\ * Correspondence should be addressed to W. Guan, Email: w.guan@psu.edu
}




\section{Supplementary Information}

\section{Supplementary Text}

\section{Gregg and Steidley particle sizing model}

Among various particle sizing models, Gregg and Steidley's expression was suitable for the case when sphere diameter is approaching to the orifice diameter. The expression was derived from the following relation,

$$
R=\rho \int \frac{d z}{A(z)}
$$

where $R$ is the resistance of the tube and $\mathrm{A}(\mathrm{z})$ is the cross-sectional area of the tube where the area does not contain the sphere. The tube is centered in the z-axis, thus particles flow toward the $\mathrm{z}$ direction. The current density across the orifice cross-section is assumed to be uniform. To incorporate the varying $A(z), A(z)=\pi D^{2} / 4$ for empty orifice and $A(z)=\frac{\pi}{4}\left(D^{2}-d^{2}+4 z^{2}\right)$ for the sphere containing orifice were substituted into the $E q$. S1 (D: diameter of orifice, $d$ : diameter of thesphere). The relative resistance changes $(\Delta R / R)$ in orifice can be expressed in terms of orifice and sphere diameters. Since the model was derived from Eq. S1, a uniform current density is desired across the cross-section of the orifice for optimal estimation (e.g., particle size comparable to the orifice diameter). Based on this rationale, we chose Gregg and Steidleye's model since our polystyrene bead diameter is similar to the sensing pore diameter. 


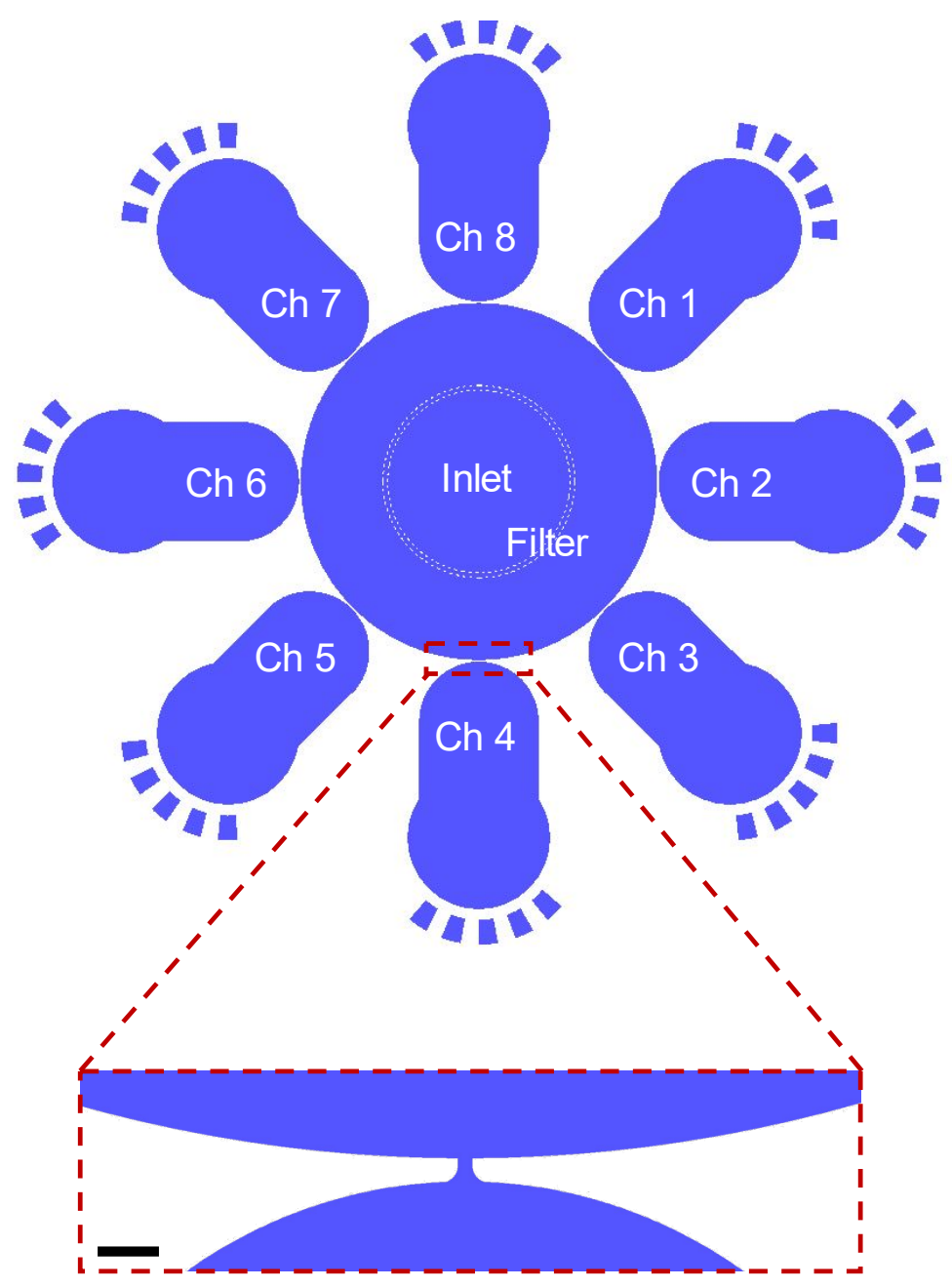

Figure S1. 8-channel microfluidic device layout. The enlarged view showed the resistive pulse sensing unit, which has micropore with width, length, and height of $18 \mu \mathrm{m}, 20 \mu \mathrm{m}$, and $35 \mu \mathrm{m}$ respectively (scale bar: $100 \mu \mathrm{m}$ ). 
(a)
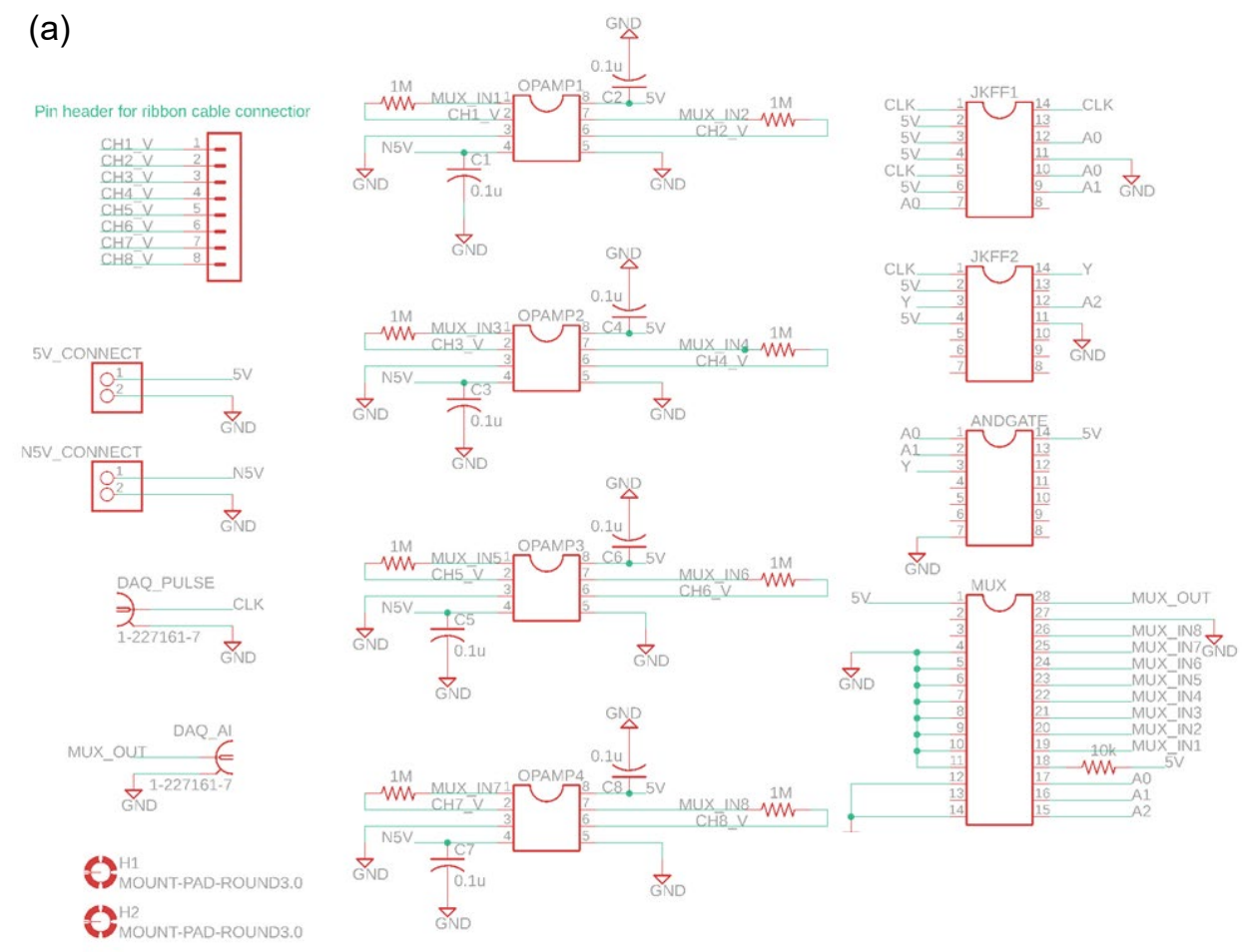

(b)

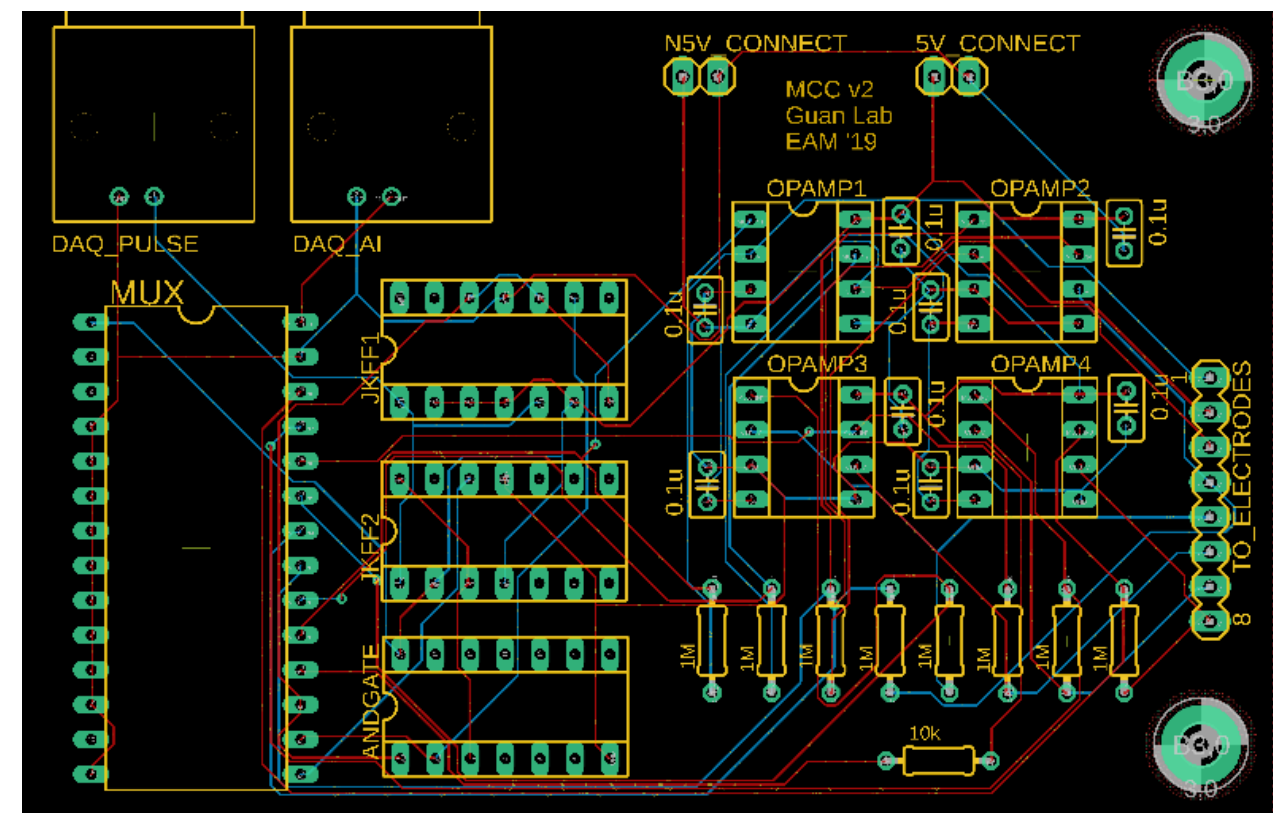

Figure S2. TDMA hardware design. (a) Electronic schematic diagram. The system includes transimpedance amplifier circuits, multiplexer, and counter circuit. DAQ provides the bias voltage for ionic current measurement and a clock signal for the 3-bit counter. External DC power source was used to provide the supply voltage for op-amps, multiplexer, JK flip-flop, and AND gate. (b) PCB layout. The PCB has dimensions of $95 \times 62 \mathrm{~mm}$ and is shielded by Faraday cage to reduce the environmental noise. 
Ch 1

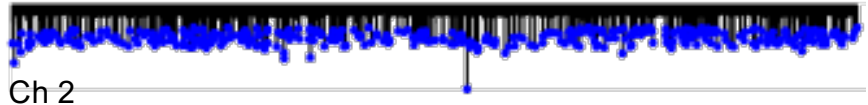

Ch 2

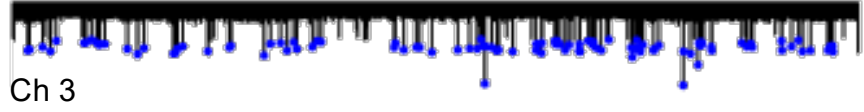
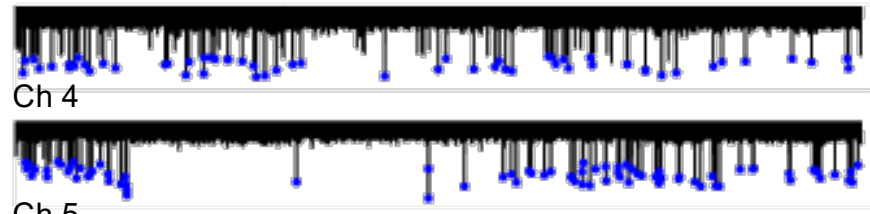

Ch 5

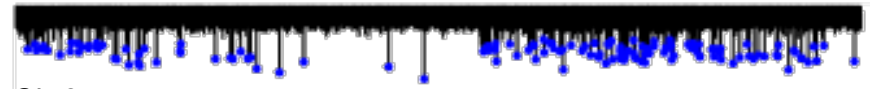

Ch 6

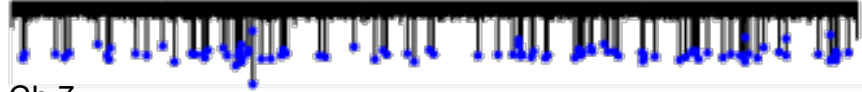

Ch 7

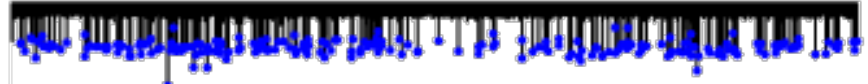

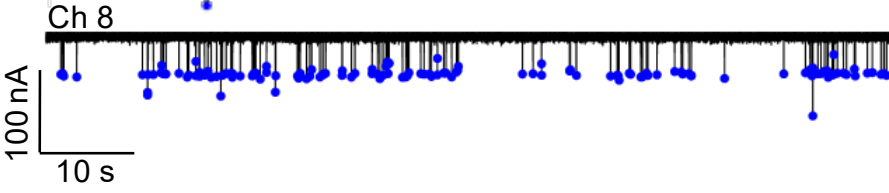

Figure S3. Validation of custom-built peak detection algorithm. The algorithm continuously finds the local minimum value and signal baseline. The current dip was calculated by subtracting local minimum value from the signal baseline. Minimum peak values for individual resistive pulses were marked by blue circles. 

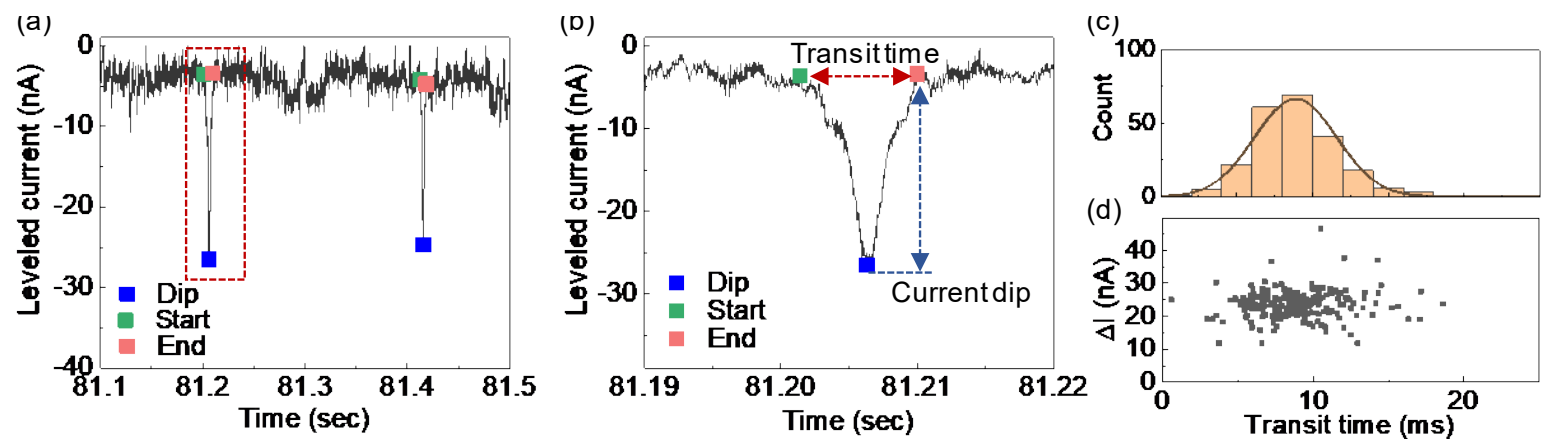

Figure S4. Time trace for the $10 \mu \mathrm{m}$ beads. (a) Representative ionic current dips (b) Enlarged the view of the current dip (red box from Figure S4a). The pulse has the transit time of $\sim 8 \mathrm{~ms}$ and current dip of $22 \mathrm{nA}$. (c) Histogram of transit time for $10 \mu \mathrm{m}$ beads. The distribution shows a mean transit time of $8 \pm 2.7 \mathrm{~ms}(N=227)$.

Table S1. Cross-correlation among different sensing channels

\begin{tabular}{|c|c|c|c|c|c|c|c|c|}
\hline $\mathbf{8}$ & -0.15 & 0.15 & -0.12 & 0.08 & -0.03 & 0.09 & -0.15 & 1.00 \\
\hline $\mathbf{7}$ & 0.15 & -0.12 & 0.14 & -0.05 & 0.22 & -0.19 & 1.00 & \\
\hline $\mathbf{6}$ & -0.08 & 0.21 & -0.06 & 0.18 & -0.23 & 1.00 & & \\
\hline $\mathbf{5}$ & 0.14 & -0.03 & 0.32 & -0.19 & 1.00 & & & \\
\hline $\mathbf{4}$ & -0.05 & 0.19 & -0.25 & 1.00 & & & & \\
\hline $\mathbf{3}$ & 0.21 & -0.16 & 1.00 & & & & & \\
\hline $\mathbf{2}$ & -0.23 & 1.00 & & & & & & \\
\hline $\mathbf{1}$ & 1.00 & & & & & & & \\
\hline Ch\# & $\mathbf{1}$ & $\mathbf{2}$ & $\mathbf{3}$ & $\mathbf{4}$ & $\mathbf{5}$ & $\mathbf{6}$ & $\mathbf{7}$ & $\mathbf{8}$ \\
\hline
\end{tabular}

